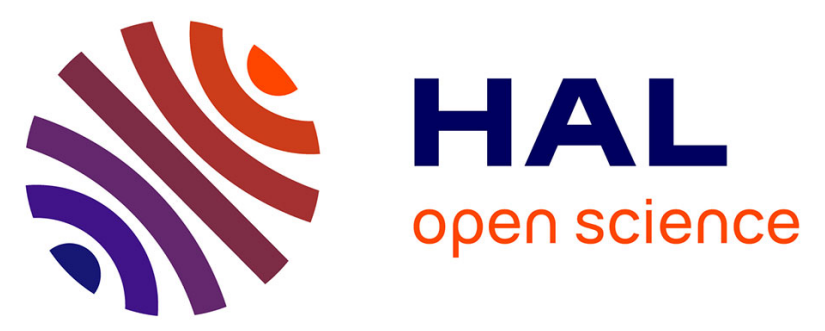

\title{
Response Time Analysis for Thermal-Aware Real-Time Systems Under Fixed-Priority Scheduling
}

Younès Chandarli, Nathan Fisher, Damien Masson

\section{To cite this version:}

Younès Chandarli, Nathan Fisher, Damien Masson. Response Time Analysis for ThermalAware Real-Time Systems Under Fixed-Priority Scheduling. IEEE 18th International Symposium on Real-Time Distributed Computing (ISORC), Apr 2015, Auckland, New Zealand. pp.84-93, 10.1109/ISORC.2015.34 . hal-01016114v2

\section{HAL Id: hal-01016114 \\ https://hal.science/hal-01016114v2}

Submitted on 20 Oct 2015

HAL is a multi-disciplinary open access archive for the deposit and dissemination of scientific research documents, whether they are published or not. The documents may come from teaching and research institutions in France or abroad, or from public or private research centers.
L'archive ouverte pluridisciplinaire HAL, est destinée au dépôt et à la diffusion de documents scientifiques de niveau recherche, publiés ou non, émanant des établissements d'enseignement et de recherche français ou étrangers, des laboratoires publics ou privés. 


\title{
Response Time Analysis for Thermal-Aware Real-Time Systems Under Fixed-Priority Scheduling
}

\author{
Younès Chandarli, Nathan Fisher, Damien Masson
}

October 20, 2015

\begin{abstract}
This paper investigates schedulability analysis for thermal-aware realtime systems. Thermal constraints are becoming more and more critical in new generation miniaturized embedded systems, e.g. medical implants. As part of this work, we adapt the PFP $P_{A S A P}$ algorithm proposed in [1] for energy-harvesting systems to thermal-aware ones. We prove its optimality for non-concrete ${ }^{1}$ fixed-priority task sets and propose a responsetime analysis based on worst-case response-time upper bounds. We evaluate the efficacy of the proposed bounds via extensive simulation over randomly-generated task systems.
\end{abstract}

\section{Introduction}

The main purpose of real-time systems is to guarantee predictable timing behavior for controlled devices. Therefore, the correctness of the results provided by such systems depends not only on the logical correctness of the output but also on the time at which it is yielded. Several formal models of real-time behavior have been proposed (e.g. task models such as sporadic, periodic, aperiodic, DAG, etc.). Prior research in real-time systems have also addressed a wide array of hardware architectures (e.g. monoprocessor, multiprocessors, memory caches, etc). However, for a new generation of real-time systems applications, e.g. medical implants, the physical environment poses additional design challenges.

One such new challenge is the necessity of managing the energy and the thermal behavior of systems. As technology scales, chips power consumption and power density are increasing rapidly. Indeed, the miniaturization of small embedded systems has allowed new real-time applications. Implantable medical devices (IMD) are an example of these new embedded systems where managing the thermal aspect is essential. IMDs are increasingly being used in medical

\footnotetext{
${ }^{1}$ I.e., we make no assumptions on tasks first release times and the initial battery level or temperature.
} 
treatments (e.g. pacemakers for heart diseases or neural implants to restore hearing/vision). However, recent studies [22, 14] have shown that the heat generated by IMDs due to the processor activity is non-negligible. Thus, designing thermal aware IMDs becomes critical as medical research has shown that a temperature increase of even $1 C^{\circ}$ can damage tissues [13] and may cause death in extreme cases [20].

Therefore, thermal-aware real-time systems must respect not only timing constraints, expressed with deadlines, but also thermal constraints which are expressed as a maximum temperature not to be exceeded. For fixed-priority real-time scheduling on monoprocessor platforms, considering this constraint requires the schedulers to add cooling periods. These additional idle times must be taken into account by scheduling algorithms and included in schedulability analysis.

Thermal-aware system design presents challenges similar to the design of energy-harvesting systems. The later collects the environmental energy to store it and use it to supply real-time systems. The real-time scheduling of these kind of systems must respect tasks deadlines without running out of energy. The similarities with thermal-aware systems come from the fact that the scheduling for energy-harvesting system has to consider a battery replenishment time (which is analogous to the cooling periods required in thermal-aware systems). In this work we use the $P F P_{A S A P}$ scheduling algorithm proposed in [1], which was proved to be optimal for non-concrete fixed-priority energy-harvesting systems, to build a reactive thermal-aware scheduling approach and an approximate schedulability analysis based on upper and lower bounds of tasks worst-case response-time.

The remainder of this paper is organized as follows. Section 2 gives a brief state of the art about thermal-aware real-time systems. Section 3 specifies and describes the model and the scope of this work. Section 4 presents the PFP $P_{A S A P}$ scheduling algorithm and its optimality for non-concrete systems. Section 5 details an approximate response-time analysis based on upper and lower bounds of tasks worst-case response time. Section 6 shows some simulations results to evaluate the effectiveness of the proposed schedulability analysis. Finally, Section 7 concludes this paper.

\section{Related Work}

In this section we give a brief overview of prior research related to thermal-aware and energy-aware real-time scheduling. Most works addressing this problem consider Dynamic Voltage and Frequency Scaling (DVFS) strategies. DVFS consists of scaling down the CPU speed and thereby lengthening task execution times to reduce energy consumption and lower the peak temperature $[11,23$, $24,25]$.

Among existing work, the proposed techniques can be divided into reactive and proactive approaches. The difference between these two approaches is that reactive schemes adapt to the temperature of the system when it reaches the 
maximum temperature or a specific trigger by switching the CPU speed or by changing scheduling decisions. In this scope, Wang et al. proposed a schedulability analysis for speed scaling scheme for frame-based task model in [25], and they completed this with a worst-case response time analysis for FIFO and fixed priority scheduling in $[24,25]$. In contrast, proactive approaches set the configuration of the system judiciously beforehand (CPU speed and scheduling decisions) so that the maximum temperature is never reached $[11,18,19]$. In this scope, Chen et al. proposed in $[10,11]$ a proactive EDF-based scheduling approach that changes the processor speed proactively by requests issued by the scheduler.

There exists also some works that address this scheduling problem without DVFS schemes by considering processors with only one frequency. In this scope, Ahmed et al. [2] proposed a technique that computes proactively the length of execution and cooling intervals so that a certain temperature is never reached. This idea was extended in [12] to support unpredictable ambient temperature fluctuations. Rehan et al. proposed in [3,4] a kind of thermal utilization of the system (using a fluid schedule) and leveraged it to obtain a necessary and sufficient conditions for systems thermal feasibility.

All the mentioned work have the following limitations:

1) Except for work in the previous paragraph [2, 3, 4, 12], all the proposed solutions rely on speed scaling to manage energy and temperature. These approaches cannot be applied to systems without DVFS capabilities.

2) Most of the scheduling solutions proposed in the literature are EDF-based. Knowing that static fixed-priority scheduling is highly used in industry, it deserves more attention and effort to study the fixed-priority real-time scheduling under thermal constraints.

Recently, some results for the scheduling problem of energy-harvesting systems, which seem to be similar to the thermal aware model, were proposed. In $[1,5,17]$ some scheduling algorithms and schedulability analysis were proposed. It consists of keeping the battery level high enough to permit task execution. The algorithm proposed in [1] behaves like a reactive approach in the thermalaware model. In this paper we use the $P F P_{A S A P}$ algorithm proposed in [1] to build a reactive approach for the thermal-aware and fixed-priority real-time systems. We propose a schedulability analysis based on tasks worst-case response time approximation techniques.

\section{Models}

\subsection{Task Model}

We consider a classical non-concrete real-time task set defined by a set of $n$ priority-ordered sporadic and independent tasks $\left\{\tau_{1}, \tau_{2}, \ldots, \tau_{n}\right\}$ where $\tau_{n}$ is the lowest priority task. Each task $\tau_{i}$ is characterized by its priority $P_{i}$, its worst-case execution time $C_{i}$, its minimum inter-arrival time $T_{i}$, its deadline $D_{i}$ and its first release time $O_{i}$. Deadlines are constrained or implicit, i.e., 


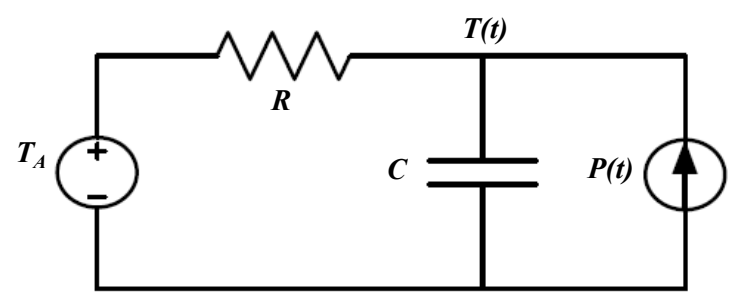

Figure 1: Thermal model

Cooling and Heating functions

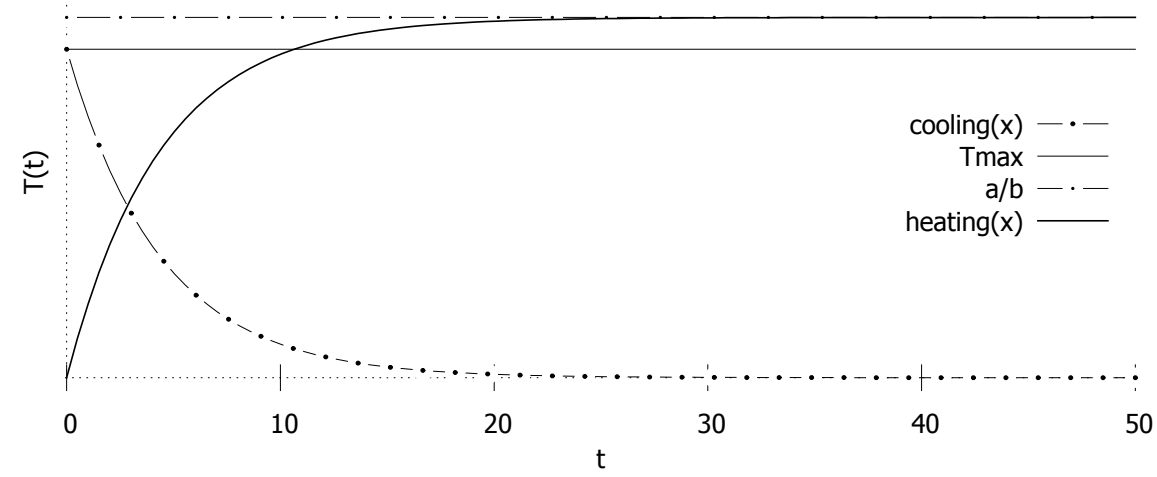

Figure 2: Cooling and Heating Functions

$\forall i, D_{i} \leq T_{i}$.

\subsection{Thermal Model}

In our model, the temperature of the system fluctuates due to heat dissipation when real-time tasks are executed on the CPU. The temperature must stay between two thresholds $T_{A}$ and $T_{\max }$ where $T_{\max }$ is the maximum tolerated temperature and $T_{A}$ is the ambient energy. The temperature of the system at time $t$ is denoted as $T(t)$. To cool down the system, we consider that tasks execution is temporarily suspended. Furthermore, we assume that the system may be in one of two states at any given time: active (i.e., heating) during which tasks may execute or inactive (i.e., cooling) during which tasks are not permitted to execute.

\subsubsection{Heating Model}

The thermal behavior of a processor can be modeled using an RC circuit [21] shown in Figure 1. In this model, the heating is modeled by the current denoted $P(t)$ passing through a thermal resistance $R$. The thermal capacitance is denoted $C$. Using this model, the derivative of the system temperature with 
respect to time can be calculated with Fourier's law [23] given by Equation 1.

$$
T^{\prime}(t)=\frac{P(t)}{C}-\frac{T(t)-T_{A}}{R \times C}
$$

The current passing through the resistance can be separated into two parts: the dynamic part $P_{D}(t)$ that evolves linearly with the processor frequency, denoted $s$, and the part corresponding to the energy leakage $P_{L}(t)$ which is a function of the temperature.

$$
\begin{aligned}
P(t) & =P_{D}(t)+P_{L}(t) \\
P_{D}(t) & =\beta_{0} s^{\alpha} \\
P_{L}(t) & =\beta_{1} T(t)+\beta_{2}
\end{aligned}
$$

Equations 2 to 4 give the formula to compute $P(t)$, where $\alpha, \beta_{0}, \beta_{1}$ and $\beta_{2}$ are system specific constants [23]. We consider only a monoprocessor with active/inactive modes; thus, during active periods, $P_{D}(t)$ is constant. Let us

denote $a=\frac{\beta_{0} s^{\alpha}}{C}, b=\frac{1}{R \cdot C}-\frac{\beta_{1}}{C}$ and scale $T(t)$ to be $T(t)-\frac{R \beta_{2}-T_{A}}{R \beta_{1}-1}$ to shift $T_{A}$ to 0 . We can now recognize in Equation 1 a classical linear differential equation:

$$
T^{\prime}(t)=a-b \times T(t)
$$

Then, the solution is given by:

$$
T(t)=\frac{a}{b}+\left(T\left(t_{0}\right)-\frac{a}{b}\right) \cdot e^{-b\left(t-t_{0}\right)}
$$

In this work, we assume that heating comes mainly from processor activity and that the heating produced by the other components of the system has negligible impact on its global thermal behavior. For this reason, the considered heating function only depends on time and constants and is not task-specific.

Recall that the parameters $a$ and $b$ are processor specific constants. Typical settings for these two variables are $b \approx 0.228$, and $a>1$ with $\alpha \approx 3$ (See [2]).

\subsubsection{Cooling Model}

During the cooling phases, the processor is inactive. In this paper, we assume for simplicity that the frequency $s$ is 0 . (However, this can easily be generalized to allow some fixed power dissipation during inactive phases). Then $a=0$ and the equation becomes:

$$
T(t)=T\left(t_{0}\right) \cdot e^{-b\left(t-t_{0}\right)}
$$

Again, the cooling function only depends on time and is not task specific. Figure 2 shows the curves of cooling and heating functions. We can see that the cooling function slows down rapidly because of the exponential function. This means that cooling for several short intervals is better for temperature and thereby for tasks response time than few and long ones. 


\section{The $P F P_{A S A P}$ algorithm}

In [1], a scheduling algorithm for energy harvesting systems called $P F P_{A S A P}$ was introduced. This algorithm is a fixed-priority one which takes into account tasks energy consumption and the battery capacity during scheduling operations for energy-harvesting systems. Tasks are executed according to their priority when the available energy is enough to execute and only replenishes the battery otherwise, jobs execution can be suspended to replenish energy as much as needed to execute at least one time unit. This algorithm was proved to be optimal for non-concrete fixed-priority energy-harvesting systems. In this section we adapt this algorithm to thermal-aware systems and we explore its optimality for the model described in Section 3.

With the thermal constraints, the behavior of $P F P_{A S A P}$ becomes as following: it executes jobs whenever the temperature is enough below $T_{\max }$ to execute at least one time unit without exceeding $T_{\max }$, then, it idles the system to cool down as long as needed to resume executions.

Below, we first address the $P F P_{A S A P}$ worst-case scenario, then we discuss its optimality from real-time scheduling point of view.

\subsection{Worst-case scenario}

The aim of this section is to prove that critical instant or the worst-case scenario for non-concrete fixed-priority thermal-aware systems is still the synchronous release of all tasks but with $T(0)=T_{\max }$.

Figure 3(a) illustrates the case where all tasks are requested simultaneously. If at least one higher priority task is requested later, the response time of lower priority tasks decreases as illustrated in Figure 3(c). Then, if higher priority tasks are requested earlier, the response time of lower priority tasks cannot be longer than the one of the synchronous scenario as shown in Figures 3(d). Furthermore, if the initial temperature of the system is lesser than $T_{\max }$, then, less cooling time is needed which leads to shorter response times for all tasks.

Theorem 1. Let $\Gamma$ denote a non-concrete task set composed of $n$ priorityordered tasks with constraint or implicit deadlines. The PFPASAP worst-case scenario for any task of $\Gamma$ occurs whenever this task is requested simultaneously with requests of all higher priority tasks and the system temperature is at the maximum level $T_{\max }$.

Proof. Let $\left\{\tau_{1}, \tau_{2}, \ldots, \tau_{n}\right\}$ be a set of $n$ priority-ordered tasks where $\tau_{n}$ is the task with the lowest priority. Let $S_{i}^{s}$ denote the scenario where task $\tau_{i}$ and all higher priority tasks are requested simultaneously at the maximum temperature $T_{\max }$. The worst-case scenario for a task $\tau_{i}$ is the one that maximizes its response time, i.e. the scenario that delays the most the termination date of the first job of the $i^{\text {th }}$ priority level.

If $S_{i}^{s}$ is not the worst scenario, there must be an other one leading to a greater response time for the $i^{\text {th }}$ priority level. 

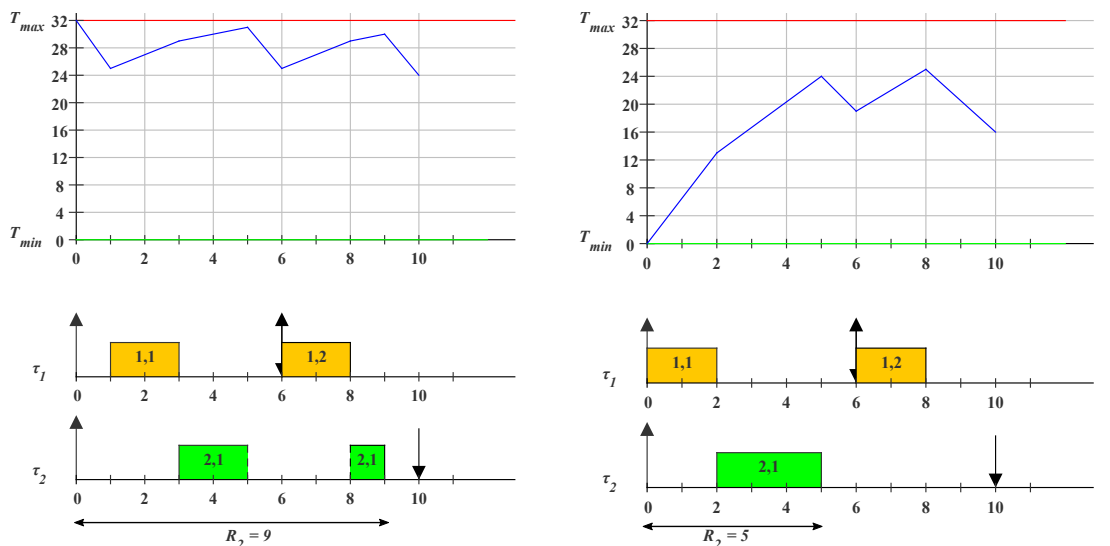

(a) Synchronous

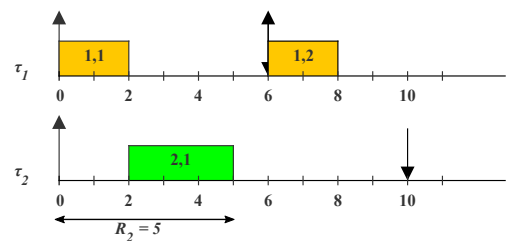

(b) $T(0)<T_{\max }$
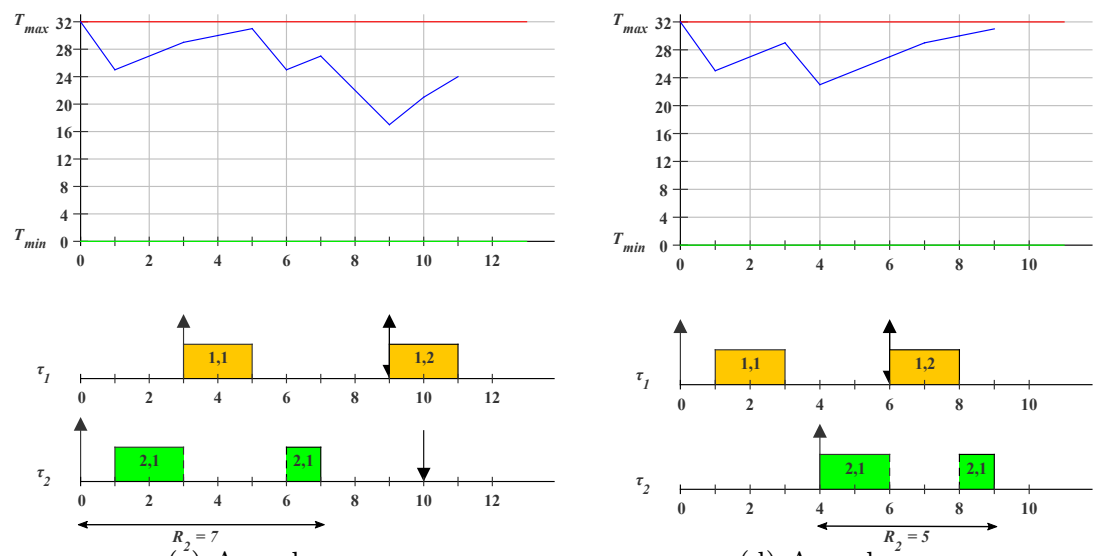

(c) Asynchronous

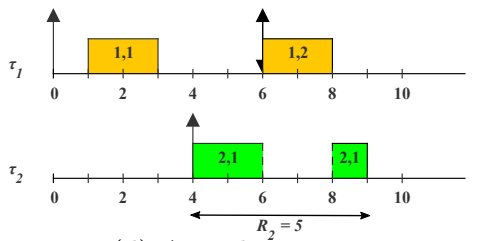

(d) Asynchronous

Figure 3: Worst-case scenario 
Firstly, we consider the scenario where $T(0)<T_{\max }$. In this case the system is not heated at the maximum. Therefore, the system needs shorter cooling time than the scenario where $T(0)=T_{\max }$, and $P F P_{A S A P}$ introduces shorter or equal cooling periods that lead to a shorter response time for all the tasks. This is in contradiction with our hypothesis, thus, such a scenario cannot lead to longer response times.

Secondly, we consider the scenario with different offsets. Let us denote $S_{i}^{a}$ as the scenario where $T(0)=T_{\max }$ and all tasks have different offsets. In this case we distinguish two possibilities:

(i) Where at least a task of higher priority than $\tau_{i}$ is requested later: knowing that all the tasks heat the system following the same pattern, i.e., by considering that the heating comes only from processor activity and that heating is faster than cooling, task $\tau_{i}$ will undergo less higher priority interferences (as in the classical critical instant proved by Liu and Layland in [15]), and then, it need less cooling to finish. Therefore, the final response time of $\tau_{i}$ is lesser than or equal to the one given by scenario $S_{i}^{s}$ which is a contradiction. Thus, such a scenario cannot lead to longer response times.

(ii) Where at least a task of higher priority than $\tau_{i}$ is requested earlier: when $\tau_{i}$ is requested later than a higher priority task, it undergoes less interference from this task because, first, a part of it was executed before $\tau_{i}$ request time, and second, the increase of temperature due to the higher priority task execution cannot be higher than $T_{\max }$, and finally, if $\tau_{i}$ is requester much later than the higher priority task, we just have shift the landscape to fit case (i). Therefore, shifting higher priority tasks request times cannot increase the response time of lower priority tasks and this scenario cannot be worse than $S_{i}^{s}$.

Therefore, in all possible situations, the response-time of a task $\tau_{i}$ is lesser or equal to the one led by a synchronous release of all higher priority tasks when the temperature is at the maximum level.

\subsection{The optimality of $P F P_{A S A P}$}

The $P F P_{A S A P}$ algorithm was proved to be optimal for the fixed-priority scheduling problem off non-concrete energy-harvesting systems [1] which is close to the same scheduling problem of thermal-aware systems. "Optimality" here means that if the optimal algorithm fails to schedule a given task set, then, no other algorithm of the same class of algorithms (i.e., processor-speed scheduling under fixed-priority) can do it.

In this subsection we extend the optimality of $P F P_{A S A P}$ to non-concrete thermal-aware systems.

Theorem 2. The PFP $P_{A S A P}$ scheduling algorithm is optimal for fixed-priority thermal-aware non-concrete task sets with constrained or implicit deadlines.

Proof. Let $\Gamma$ denote a non-concrete task set. We suppose that $\Gamma$ is feasible using a fixed-priority assignment, but not schedulable with $P F P_{A S A P}$ using the same priority assignment. This means that at least one task denoted as $\tau_{k}$ misses its 
deadline in the worst-case scenario (see Theorem 1). Indeed, it is sufficient to consider only the first job because the deadlines are constrained wich means that there is no overlapping with two jobs of the same task. According to PFP $P_{A S A P}$ rules, a deadline miss can occur in the worst-case scenario only in two cases: 1) the workload ${ }^{2}$ is greater than the available time, 2) the workload plus the accumulated cooling time is greater than the available time.

1 ) if the workload from the critical instant (time 0) to time $D_{k}$, the first deadline of $\tau_{k}$, is alone greater than time interval $\left[0, D_{i}\right]$, then, it is obviously impossible to schedule the first job of $\tau_{k}$ and higher priority jobs without missing $D_{k}$, this is not possible even without thermal constrains because the available time is not sufficient to schedule all the workload within $\left[0, D_{k}\right]$. Then, in this case the task set cannot be feasible with any algorithm and the supposed algorithm cannot exist.

2 ) if a deadline is missed with $P F P_{A S A P}$ even though the workload is lesser than the available time, then, this means that the workload of time interval $\left[0, D_{k}\right]$ and the needed cooling time is greater than the available time, i.e $D_{k}$ time units. Knowing that the cooling periods produced by $P F P_{A S A P}$ are as long as needed to execute at least one time unit, this means that they are as short as possible. Furthermore, we know that the cooling function is exponentially decreasing (See Equation 7), then, the shorter cooling periods are, the shorter the total needed cooling time is. This is true because the longer cooling is, the less efficient it is, as we mentioned in Section 3. More formally, cooling $x$ times one time unit decreases more the temperature than only one cooling period of length $x$ time units; observe that $e^{-b x} \leq x e^{-b}$ for all $x \geq 1$. Thus, Equation 7) implies

$$
T(0)-T(0) \cdot e^{-b \cdot x} \geq T(0)-x \times T(0) \cdot e^{-b(1-0)}
$$

Therefore, any other schedule than $P F P_{A S A P}$ 's one has necessarily cooling periods of same length or longer, then, the response time of $\tau_{k}$ produced by the supposed algorithm is necessarily greater than $D_{k}$. Thus, in this case, no other algorithm can schedule this task set.

Then, we prove that $P F P_{A S A P}$ is optimal for non-concrete fixed-priority thermal-constrained task sets with constrained or implicit deadlines.

\section{Response-Time Analysis}

This section provides a response-time analysis for the schedule produced by the optimal algorithm $P F P_{\text {asap }}$ in the worst-case scenario, i.e., the synchronous release of all the tasks when $T(0)=T_{\max }$. We discuss the difficulty of an exact analysis and then we propose an approximate one.

\footnotetext{
${ }^{2}$ the execution time of the considered task and the ones of higher priority tasks
} 


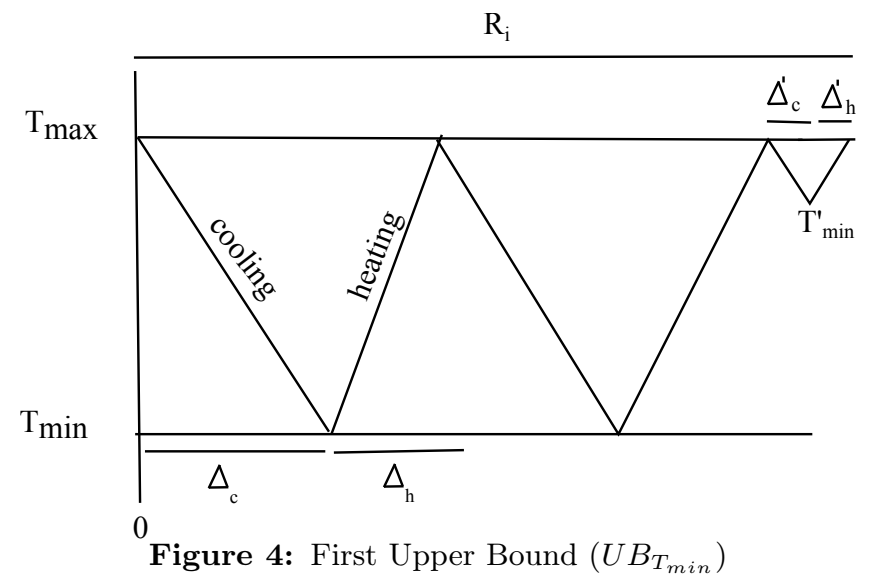

\subsection{Exact Analysis}

The exact analysis provides the exact response time of all tasks. Thus, it must estimate accurately the length of all cooling and heating periods.

However, this cannot be done with a generic equation because due to the discrete time, all cooling periods are not of the same length in the actual schedule. Furthermore, without the effective values of parameters, it is hard to estimate the order and the number of long and short cooling periods which have a significant impact on the response time value. Therefore, the remaining way to get an exact analysis is to simulate the schedule of $P F P_{A S A P}$ in the worst-case scenario and compute the response time of the first job of each task.

\subsection{Approximate Analysis}

The aim of this work is to propose a schedulability analysis for thermal-aware real-time systems. Such an analysis must consider not only the processor workload but also the additional cooling time needed to respect the thermal constraints. To cope with the difficulty of providing an exact analysis, one can propose an approximate one that can be only sufficient instead of necessary and sufficient. This can be achieved by upper bounding tasks worst-case response time produced by $P F P_{A S A P}$ algorithm.

\subsubsection{First Upper bound $\left(U B_{T_{m i n}}\right)$ :}

Knowing that $P F P_{A S A P}$ cools down the system enough to execute at least one time unit, cooling periods are as short as possible. Furthermore, we know also that the cooling function is exponentially decreasing and the heating is asymptotically increasing, then, one can lengthen jobs response times by putting the cooling units together and the heating ones together such that $T_{\max }$ is never exceeded. By doing so, the cooling slows down after a while and the system needs more time to cool down, and heating becomes faster which heats up the system in a shorter amount of time. 
Description The upper bound of task $\tau_{i}$ worst-case response time according to $U B_{T_{m i n}}$ is described by Figure 4 . It consists of:

- cooling down the system from $T_{\max }$ to $T_{\min }$, where $T_{\min }>T_{A}{ }^{3}$

- a ceiling function applied to the time from $T_{\max }$ to $T_{\min }$ to ensure an integer number of time units (this is safe since it only over estimates the time required to reach $T_{\text {min }}$ ),

- executing jobs and heating up the system until $T_{\max }$ is reached or there is no pending workload,

- repeating this cycle of cooling-heating until there is no pending workload,

- the last cycle may be shorter because of the remaining workload which is shorter than a full cycle. The corresponding cooling time is adjusted.

The response time upper bound of task $\tau_{i}$ of priority level- $i$ that is requested simultaneously with higher priority tasks with $T(0)=T_{\max }$ is given by Equation 9

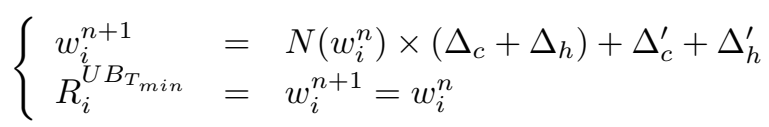

where :

- $N(w)$ is the number of full cooling-heating cycles needed to execute the workload $w$ without exceeding $T_{\max }$ :

$$
N(w)=\left\lfloor\frac{w}{\Delta_{h}}\right\rfloor
$$

- $w$ is the workload of time interval $\left[0, w_{i}^{n}\right]$ :

$$
w=\sum_{j \leq i}\left\lceil\frac{w_{i}^{n}}{T_{j}}\right\rceil \times C_{j}
$$

- $\Delta_{h}$ is the time to execute jobs and heat up the system from $T_{\min }$ to $T_{\max }$. (Obtained by solving Equation 6):

$$
\Delta_{h}=\left\lfloor\frac{\ln \left(\frac{b \cdot T_{\min }-a}{b \cdot T_{\max }-a}\right)}{b}\right\rfloor
$$

- $\Delta_{c}$ is the time to cool down the system from $T_{\max }$ to $T_{\min }$. (Obtained by solving Equation 7):

$$
\Delta_{c}=\left\lceil\frac{\ln \left(T_{\max }\right)-\ln \left(T_{\min }\right)}{b}\right\rceil
$$

\footnotetext{
${ }^{3}$ Observe that a low $T_{\min }$ value may result in an extremely pessimistic upper bound due to the nature of the cooling function; It decreases asymptotically to $T_{A}$, so waiting until $T_{m i n}$ is too pessimistic because of the nature of the cooling function.
} 


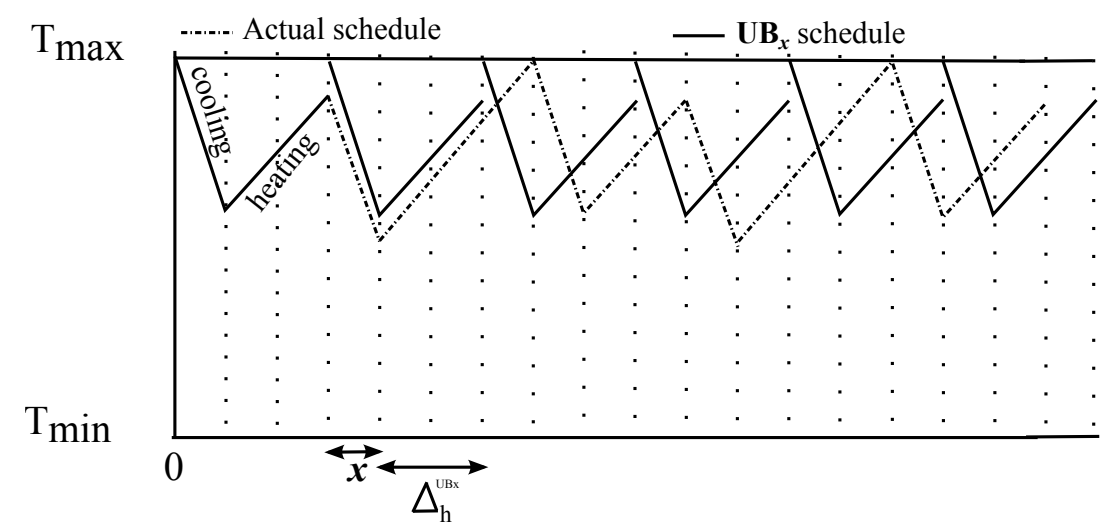

Figure 5: Parametric Upper Bound $U B_{x}$

- $\Delta_{h}^{\prime}$ is the remaining execution time of the busy period:

$$
\Delta_{h}^{\prime}=w-N\left(w_{i}^{n}\right) \times \Delta_{h}
$$

- $\Delta_{c}^{\prime}$ is the cooling time needed for $\Delta_{h}^{\prime}$ :

$$
\Delta_{c}^{\prime}=\left\lceil\frac{\ln \left(T_{\max }\right)-\ln \left(T_{\min }^{\prime}\right)}{b}\right\rceil
$$

- $T_{\min }^{\prime}$ is the maximum temperature needed to execute the remaining part of the workload $\Delta_{h}^{\prime}$ without exceeding $T_{\max }$ :

$$
T_{\min }^{\prime}=\left(T_{\max }-a / b\right) e^{b \Delta_{h}^{\prime}}+a / b
$$

Theorem 3. An upper bound on the worst-case response time for task $\tau_{i}$ in the worst-case scenario described in Section 4.1 can be obtained from a sequence of execution units of higher priority jobs and the necessary cooling time units where the cooling periods are as long as needed to cool down the system from $T_{\max }$ to $T_{\min }\left(T_{\min }>0\right)$ and the heating periods are as long as needed to reach $T_{\text {max }}$ starting from $T_{\text {min }}$, as described by Equation 9.

Proof. Due to space limitation, the proof is not included in the paper. However, it can be found in the technical report [9].

\subsubsection{Parametric Upper bound $\left(U B_{x}\right)$ :}

Description The idea of this upper bound is to keep the same behavior as the $P F P_{A S A P}$ algorithm by cooling down for some time units and then executing jobs until reaching $T_{\max }$. The approximation comes from the fact that time is discrete and that cooling periods are of a fixed length $x$ (where $x \in \mathbb{N}^{*}$ ) instead of the minimum length needed to execute at least one time unit. Then, the execution or the heating periods may not reach $T_{\max }$ in an integer number of 
time units. Thus, we consider only the integer part of heating periods (with floor function) and that $T_{\max }$ is exactly or nearly reached at the end of each heating period which adds additional cooling time than the actual schedule. Figure 5 describes the scenario used to obtain $U B_{x}$. It consists of:

- Cooling down the system for $x$ time units, where $x$ a is positive integer that must be greater or equal to $\Delta_{c}^{U B_{x}}$ the minimum time needed to decrease the temperature such that the system can execute at least one time unit, i.e. $x \geq \Delta_{c}^{U B_{x}}$. Equation 17 computes $\Delta_{c}^{U B_{x}}$; the ceiling function is used to respect the discrete time and to ensure that the system is enough cold to execute at least one time unit.

$$
\Delta_{c}^{U B_{x}}=\left\lceil\frac{\ln \left(\frac{b T_{\max }}{\left(b T_{\max }-a\right) e^{b}+a}\right)}{b}\right\rceil
$$

- Then, executing jobs and heating up the system until $T_{\max }$ is reached (without exceeding) or there is no pending workload. The length of this period is an integer. The floor function is used to ensure not exceeding $T_{\max }$.

- Repeating the cooling-heating cycles until there is no pending workload.

- The length of the last cooling period is still the same even if the remaining workload is smaller.

The upper bound according to $U B_{x}$ of task $\tau_{i}$ of priority level- $i$ that is requested simultaneously with higher priority tasks with $T(0)=T_{\max }$ is given by Equation 18.

$$
\left\{\begin{array}{l}
w_{i}^{n+1}=N\left(w_{i}^{n}\right) \times x+w \\
R_{i}^{U B_{x}}=w_{i}^{n+1}=w_{i}^{n}
\end{array}\right.
$$

where :

- $N(w)$ is the number of cooling periods needed to execute the workload $w$ without exceeding $T_{\max }$ :

$$
N(w)=\left\lceil\frac{w}{\Delta_{h}^{U B_{x}}}\right\rceil
$$

- $w$ is the workload of time interval $\left[0, w_{i}^{n}\right]$ :

$$
w=\sum_{j \leq i}\left\lceil\frac{w_{i}^{n}}{T_{j}}\right\rceil \times C_{j}
$$


- $\Delta_{h}^{U B_{x}}$ is the time to execute jobs and heat up the system from the temperature reached after $x$ time units of cooling to $T_{\max }$ :

$$
\Delta_{h}^{U B_{x}}=\left\lfloor\frac{\ln \left(\frac{b \cdot T_{\max } \cdot e^{-b \cdot x}-a}{b \cdot T_{\max }-a}\right)}{b}\right\rfloor
$$

We choose cooling periods longer or equal to $\Delta_{c}^{U B_{x}}$, i.e. $x \geq \Delta_{c}^{U B_{x}}$, because it is sufficient to execute at least one time unit without exceeding $T_{\max }$ which is close the behavior of $P F P_{A S A P}$ algorithm.

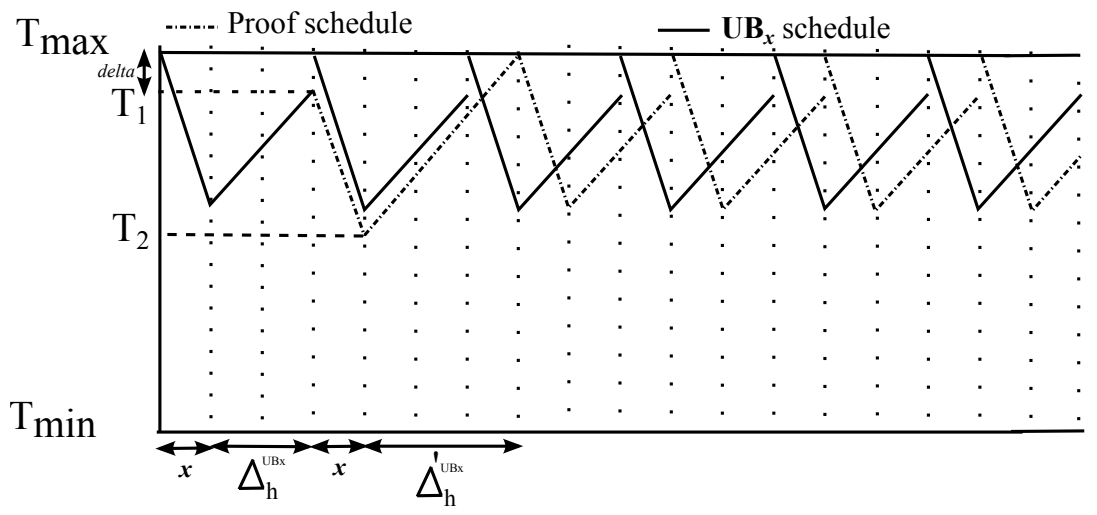

Figure 6: $U B_{x}$ proof insight

To prove that $U B_{x}$ upper bounds the actual response time, we first check the case when $x=\Delta_{c}^{U B_{x}}$. We know that in the actual schedule, the accumulation of the temperature gained at the end of each heating period, due to the discrete time, is lesser than $T_{\max }$. We denote the gap $\delta$.Thus, this accumulated temperature can be used at least by one heating period which is supposed to be longer as shown in Figure 6. Then, we compare the length of the new heating period $\Delta_{h}^{\prime}$ (See Equation 20) to the one of $U B_{x}$, i.e. $\Delta_{h}^{U B_{x}}$, and the total number of cooling/heating produced by $U B_{x}$, i.e., $N^{U B_{x}}(w)$, and the one produced by the actual schedule denoted $N^{\prime}(w)$.

$$
\begin{aligned}
& \left\{\begin{array}{l}
T_{\max }=\left(a+\left(b \cdot T_{2}-a\right) e^{-b \cdot \Delta_{h}^{\prime}}\right) / b \\
T_{2}=\left(T_{\max }-\delta\right) e^{-b \cdot x}
\end{array}\right. \\
& \Delta_{h}^{\prime}=\left\lfloor\frac{\ln \left(\frac{b \cdot\left(T_{\max }-\delta\right) \cdot e^{-b \cdot x}-a}{b \cdot T_{\max }-a}\right)}{b}\right\rfloor
\end{aligned}
$$

Lemma 1. For $x=\Delta_{c}^{U B_{x}}$, each heating interval of the actual schedule $\Delta_{h}^{\prime}$ given by Equation 20 is greater or equal than $U B_{x}$ 's ones, i.e. $\Delta_{h}^{U B_{x}} \leq \Delta_{h}^{\prime}$. 
Proof. Let's suppose that $\Delta_{h}^{U B_{x}}>\Delta_{h}^{\prime}$, then:

$$
\begin{aligned}
& \Delta_{h}^{U B_{x}}>\Delta_{h}^{\prime} \Rightarrow \\
& \left\lfloor\frac{\ln \left(\frac{b \cdot T_{\max } \cdot e^{-b x}-a}{b T_{\max }-a}\right)}{b}\right\rfloor>\left\lfloor\frac{\ln \left(\frac{b\left(T_{\max }-\delta\right) e^{-b x}-a}{b T_{\max }-a}\right)}{b}\right\rfloor \\
& \Rightarrow \frac{\ln \left(\frac{b T_{\max } e^{-b x}-a}{b T_{\max }-a}\right)}{b}>\frac{\ln \left(\frac{b\left(T_{\max }-\delta\right) e^{-b x}-a}{b T_{\max }-a}\right)}{b} \\
& \Rightarrow \frac{b T_{\max } e^{-b x}-a}{b T_{\max }-a}>\frac{b\left(T_{\max }-\delta\right) e^{-b x}-a}{b T_{\max }-a}
\end{aligned}
$$

Knowing that $b T_{\max }<a$, then:

$$
\begin{aligned}
\Delta_{h}^{U B_{x}}>\Delta_{h}^{\prime} & \Rightarrow b T_{\max } e^{-b x}-a<b\left(T_{\max }-\delta\right) e^{-b x}-a \\
& \Rightarrow \delta<0
\end{aligned}
$$

Contradiction because $b<1, \delta \geq 0$ and $b \cdot T_{\max }<a$. Therefore, we prove by contradiction that $\Delta_{h}^{U B_{x}} \leq \Delta_{h}^{\prime}$

Lemma 2. For $x=\Delta_{c}^{U B_{x}}$, the number of cooling periods produced by a PF $P_{A S A P}$ actual schedule denoted $N^{\prime}(w)$ is lesser or equal to the ones produced by $U B_{x}$, i.e. $N^{\prime}(w) \leq N^{U B_{x}}(w)$.

Proof. Let's suppose that $N^{\prime}(w)>N^{U B_{x}}(w)$. From Lemma 1 we know that $\Delta_{h}^{U B_{x}} \leq \Delta_{h}^{\prime}$ at least for one time, then:

$$
N^{\prime}(w)=\left\lceil\frac{w-\Delta_{h}^{\prime}}{\Delta_{h}^{U B_{x}}}\right\rceil+1=\left\lceil\frac{w-\left(\Delta_{h}^{U B_{x}}+\delta\right)}{\Delta_{h}^{U B_{x}}}\right\rceil+1
$$

where $\delta \geq 0$. Then, $N^{\prime}(w)$ can be written as following:

$$
N^{\prime}(w)=\left\lceil\frac{w-\delta}{\Delta_{h}^{U B_{x}}}\right\rceil
$$

Therefore, if $N^{\prime}(w)>N^{U B_{x}}(w)$, then:

$$
\begin{aligned}
N^{\prime}(w)>N^{U B_{x}}(w) & \Rightarrow\left\lceil\frac{w-\delta}{\Delta^{U B_{x}}}\right\rceil>\left\lceil\frac{w}{\Delta_{h}^{U B_{x}}}\right\rceil \\
& \Rightarrow \frac{w-\delta}{\Delta_{h}^{U B}}>\frac{w}{\Delta_{h}^{U B_{x}}} \\
& \Rightarrow \delta<0
\end{aligned}
$$

Contradiction, because $\Delta_{h}^{\prime} \geq \Delta_{h}^{U B_{x}}$ and $\delta \geq 0$. Therefore, we prove that $N^{\prime}(w) \leq N^{U B_{x}}(w)$

Theorem 4. An upper bound on the worst-case response time for task $\tau_{i}$ in the worst-case scenario described in Section 4.1 can be obtained from a sequence of execution units of $\tau_{i}$ and those of higher priority jobs and the necessary cooling time units where the cooling periods are of $x$ time units and the heating periods are integers and as long as needed to reach $T_{\max }$ (without exceeding) after $x$ time units of cooling, as described by Equation 18. 
Proof. To prove this theorem, we have to first solve the case where $x=\Delta_{c}^{U B_{x}}$, and the one where $x>\Delta_{c}^{U B_{x}}$.

Case where $x=\Delta_{c}^{U B_{x}} \quad$ From Lemma 1 and Lemma 2 we know that $N^{\prime}(w) \leq$ $N^{U B_{x}}(w)$, then:

$$
\begin{aligned}
N^{\prime}(w) \leq N^{U B_{x}}(w) & \Rightarrow N^{\prime}\left(w_{i}^{n}\right) x+w_{i}^{n} \leq N^{U B_{x}}\left(w_{i}^{n}\right) x+w_{i}^{n} \\
& \Rightarrow w_{i}^{\prime} \leq w_{i}^{U B_{x}} \Rightarrow R_{i}^{\prime} \leq R_{i}^{U B_{x}}
\end{aligned}
$$

Hence, when $x=\Delta_{c}^{U B_{x}}, U B_{x}$ is an upper bound of tasks worst-case response time according to $P F P_{A S A P}$ algorithm.

Case where $x>\Delta_{c}^{U B_{x}}$ Lengthening cooling periods by increasing the $x$ parameter is expected to increase the pessimism of $U B_{x}$ by increasing tasks response time over estimation given by Equation 18. To prove that, one can check if the $U B_{x}$ 's response time computation function is increasing. From Equation 18, the response time function can be written as follows:

$$
w_{i}^{n+1}=\left\lceil\frac{w}{\left\lfloor\frac{\ln \left(\frac{b \cdot T_{\max } \cdot e^{-b \cdot x}-a}{b \cdot \operatorname{Tmax}_{\operatorname{ma}}-a}\right)}{b}\right\rfloor}\right\rfloor \times x+w
$$

Recall that the ceil function is used to ensure a non null integer length for cooling period, and that floor function is used ensure never exceeding $T_{\max }$ after a heating period. Even though, these two functions contribute to increase the pessimism of $U B_{x}$, removing them does not change the response time function monotonicity. Then, we can study the monotonicity of this new function that we call $f(x)$ by computing its derivative function as follows:

$$
\begin{gathered}
f(x)=\frac{w \cdot b \cdot x}{\ln \left(\frac{b \cdot T_{\max } \cdot e^{-b \cdot x}-a}{b \cdot T_{\max }-a}\right)}+w \\
f^{\prime}(x)=\frac{b \cdot \ln \left(\frac{b \cdot T_{\max } \cdot e^{-b \cdot x}-a}{b \cdot T_{\max }-a}\right)+\frac{b^{3} \cdot T_{\max } \cdot x \cdot e^{-b \cdot x}}{b \cdot T_{\max } \cdot e^{-b \cdot x}-a}}{\left(\ln \left(\frac{b \cdot T_{\max } \cdot e^{-b \cdot x}-a}{b \cdot T_{\max }-a}\right)\right)^{2}}
\end{gathered}
$$

Due to the lack of space, we do not show the whole study of $f(x)$ 's sign, we do this only with deductions. Thus, the sign of $f^{\prime}(x)$ depends only on the numerator part of the fraction, we denote this part $g(x)$. Then, knowing that $g(0)=$ $\ln \left(a /\left(a-b T_{\max }\right)\right)>0$, we can say that $g(x)$ is positive in interval $[1,+\infty[$ because first the left part (the logarithm part) is positive, because of the logarithm function, and increasing, due to to the reverse exponential function; and second the right part is negative, because $b \cdot T_{\max }<a$, and slightly decreasing from 


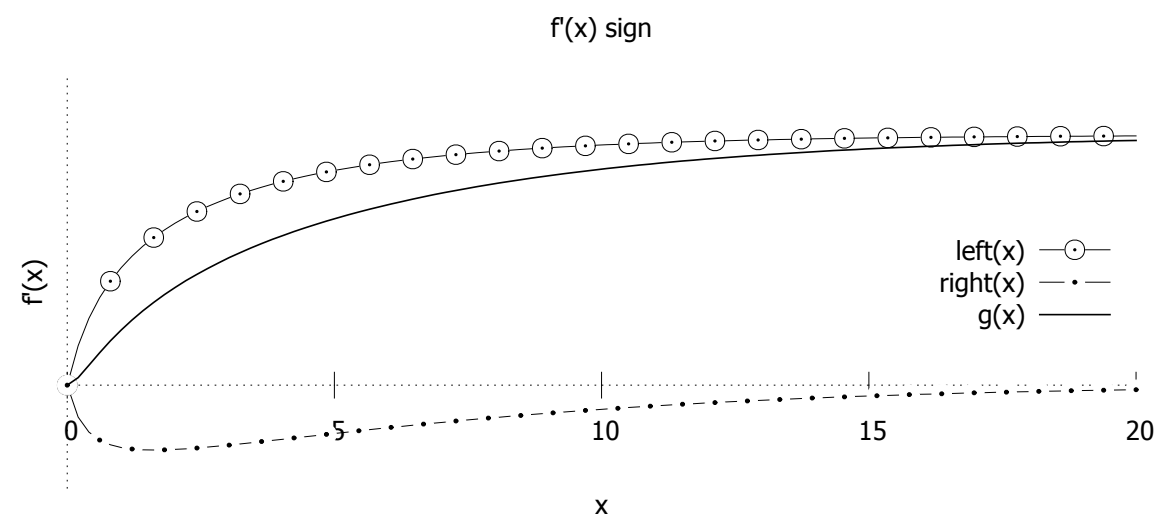

Figure 7: $f^{\prime}(x)^{\prime}$ 's sign

0 for a while and then it increases asymptotically to 0 as shown in Figure 7. Therefore, $f^{\prime}(x)$ is positive which means that $f(x)$ is increasing in interval $\left[1,+\infty\left[\right.\right.$, and then tasks response time according to $U B_{x}$ increases when $x$ is increasing.

Therefore we prove that $U B_{x}$ upper bounds the actual $P F P_{A S A P}$ worst-case response time.

\subsubsection{Lower bound $\left(L B_{x=1}\right)$ :}

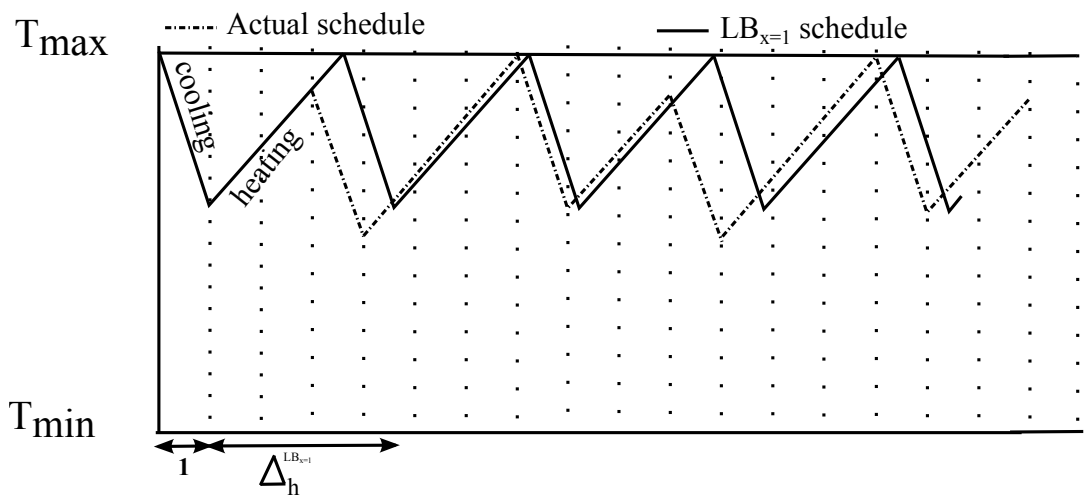

Figure 8: Lower Bound $\left(L B_{x=1}\right)$

Knowing that the actual schedule respects the discrete time constraint, we can compute a lower bound of the actual tasks worst-case response time by violating this constraint, i.e., allowing non-discrete execution times (See 8). The following points summarize the behavior of $L B_{x=1}$ :

- Cooling down the system for one time unit. This is sufficient because continous time allows executing less than one time unit. 
- Then, executing jobs and heating up the system until $T_{\max }$ is reached or there is no pending workload; this length of period is not necessarily integer.

- Repeat this cycle cooling-heating until there is not pending workload

The lower bound of task $\tau_{i}$ of priority level- $i$ that is requested simultaneously with higher priority tasks with $T(0)=T_{\max }$ is given by Equation 21

$$
\begin{cases}w_{i}^{n+1} & =N\left(w_{i}^{n}\right)+w \\ R_{i} & =w_{i}^{n+1}=w_{i}^{n}\end{cases}
$$

where :

- $N(w)$ is the number of cooling periods needed to execute the workload $w$ without exceeding $T_{\max }$ :

$$
N(w)=\left\lceil\frac{w}{\Delta_{h}^{L B_{x=1}}}\right\rceil
$$

- $w$ is the workload of time interval $\left.] 0, w_{i}^{n}\right]$.

- $\Delta_{h}^{L B_{x=1}}$ is the time to execute jobs and heat up the system from the temperature reached after one time unit of cooling to exactly $T_{\max }$ :

$$
\Delta_{h}^{L B_{x=1}}=\frac{\ln \left(\frac{b \cdot T_{\max } \cdot e^{-b}-a}{b \cdot T_{\max }-a}\right)}{b}
$$

Conjecture 1. A lower bound on the worst-case response time for task $\tau_{i}$ in the worst-case scenario described in Section 4.1 can be obtained from a sequence of execution units of $\tau_{i}$ and higher priority jobs and the necessary cooling time units where the cooling periods are of one time unit and the heating periods are continuous (not necessarily integers) and as long as needed to reach exactly $T_{\max }$ after one time unit of cooling, as described by Equation 21.

We do not prove the conjecture due to space limitation.

\subsection{4 $U B_{T_{\min }}$ vs. $U B_{x}$}

The tightness of the upper bound $U B_{x}$ relative to $U B_{T_{\min }}$ depends on the parameter $x$. In fact, the greater $x$ is, the more pessimistic $U B_{x}$ is because of the nature of the cooling function which is asymptotically decreasing to $T_{A}$. Then, for small values of $x, U B_{x}$ is tighter and for great values $U B_{T_{m i n}}$ is. The experiments presented in Section 6 demonstrates the differences in practice between $U B_{x}$ and $U B_{T_{m i n}}$ in term of tightness and complexity. 


\subsubsection{Utilization bound}

Under thermal constraints, cooling periods are needed to prevent the system to exceed $T_{\max }$. This means that for a certain processor utilization, which determines the time in which the processor is occupied, more time is needed for cooling which means that the processor cannot be used at 100\%. One can use this idea to propose a new maximum processor utilization that can respect the thermal constraints. In the following we discuss utilization bounds that consider cooling time.

Maximum utilization To compute the maximum supportable processor utilization that takes into account cooling time, one can use the idea of over estimating response times, by over estimating the cooling time needed to execute the workload of one hyper-period. We can use for instance the idea of $U B_{x}$ to compute an upper bound for the maximum supportable processor utilization.

Lemma 3. An upper bound of the processor utilization $U=\sum_{1 \leq i \leq n} C_{i} / T_{i}$ for thermal-aware fixed-priority task sets can be obtained by Equation 24.

$$
U \leq \frac{\Delta_{h}^{U B_{x}}}{\Delta_{h}^{U B_{x}}+x}
$$

Proof. We first upper bound the workload of one hyper period with $U B_{x}$ then we compute the corresponding processor utilization, and finally we compute the maximum achievable utilization. The workload of an hyper-period $L$ can be obtained by multiplying $L$ by the processor utilization $U$. Then, we can replace $w$ by $U \cdot L$ in Equation 18 to compute the time needed (cooling + workload) to satisfy the workload $U \cdot L$. Finally, we can compute the new utilization $u^{*}$, that considers cooling time, by dividing the time demand (cooling + workload) by the available time $L$, Equation 25 shows how to compute $U^{*}$.

$$
U^{*}=\frac{\left\lceil\frac{U \cdot L}{\Delta_{h}^{U B_{x}}}\right\rceil \cdot x+U \cdot L}{L}
$$

For a task set to be feasible, the new utilization $U^{*}$ must be lesser than 1 because the available time must be greater or equal to the time demand. Then,

$$
\begin{aligned}
U^{*} \leq 1 & \Rightarrow \frac{\left[\frac{U \cdot L}{\Delta_{h}^{U} B_{x}}\right] \cdot x+U \cdot L}{L} \leq 1 \\
& \Rightarrow \frac{U \cdot L \cdot x}{\Delta_{h}^{U B}+U \cdot L} \leq 1 \\
& \Rightarrow U \cdot\left(\frac{x}{\Delta_{h}^{U B_{x}}}+1\right) \leq 1 \\
& \Rightarrow U \leq \frac{\Delta_{h}^{U B_{x}}}{\Delta_{h}^{U B B x}+x}
\end{aligned}
$$


Liu and Layland bound We can use the same reasoning as the above utilization upper bound to propose a sufficient feasibility test based on Liu and Layland bound for Rate Monotonic priority assignment. In fact, we can compare the total time utilization (processor and cooling) to Liu and Layland bound.

Lemma 4. An upper bound of the processor utilization $U=\sum_{1 \leq i \leq n} C_{i} / T_{i}$ for thermal-aware fixed-priority task sets with implicit deadlines and Rate Monotonic priority assignment can be obtained by Equation 26.

$$
U \leq \frac{\Delta_{h}^{U B_{x}} \cdot n(\sqrt[n]{2}-1)}{\Delta_{h}^{U B_{x}}+x}
$$

The proof of the above lemma is not included as the steps are similar to Lemma 3.

\section{Performance Evaluation}

\subsection{Taskset generation}

To evaluate the effectiveness of our proposed schedulability test, we perform an empirical investigation. We randomly generated 100000 task sets, varying the processor utilization. We varied $U$ in the range $[0.05,1]$ in steps of 0.05 . Hence we obtained 5000 distinct task sets for each $U$ step. Each tasks set comprised 10 tasks. The thermal parameters was set as following, $T_{\max }=32$ $C^{\circ}, b=0.228$, and $a=\beta_{0} \cdot S^{3}=8$. These parameters are the ones of the whole system (including an eventual cooling device) and correspond to a classical Intel Pentium processor parameters [12]. The task parameters were randomly generated as follows: task processor utilization $\left(U_{i}=C_{i} / T_{i}\right)$ using the $U$-Unifast Discard algorithm [7], and periods randomly generated between 2 and 25200 time units with a hyper-period limitation technique [16]. Task deadlines were implicit.

We used YARISS as a simulation environment [8] which respects the following hypotheses: discrete time (all scheduling operations are performed before or after one time unit), the heating behavior follows the Fourier's law (See Equation 6 ) and temperature values are real numbers.

\subsection{Schedulability tests investigated}

We investigated the performance of the following schedulability tests.

$S I M$ : is an empirical necessary and sufficient test based on simulating the schedule of $P F P_{A S A P}$ over more than one hyper-period, starting with synchronous release and the maximum temperature level which corresponds to the worst-case scenario discussed in Section 4.1.

$U B_{T_{\min }}$ : the sufficient test presented in Section 5.2.1, we consider that $T_{\min }=1 C^{\circ}$.

$U B_{x}$ : the sufficient test presented in Section 5.2.2, the parameter $x$ is varied from 1 to 18 . 
$L B_{x=1}$ : the necessary test presented in Section 5.2.3.

$C F P$ : the exact test for fixed priority ignoring thermal and energy constraints. This was used to provide a schedulability bound, considering only processing time.

UTZ: the necessary condition described in Section 5.2.5.

LnL: the sufficient condition described in Section 5.2.5.

\subsection{Experiments}

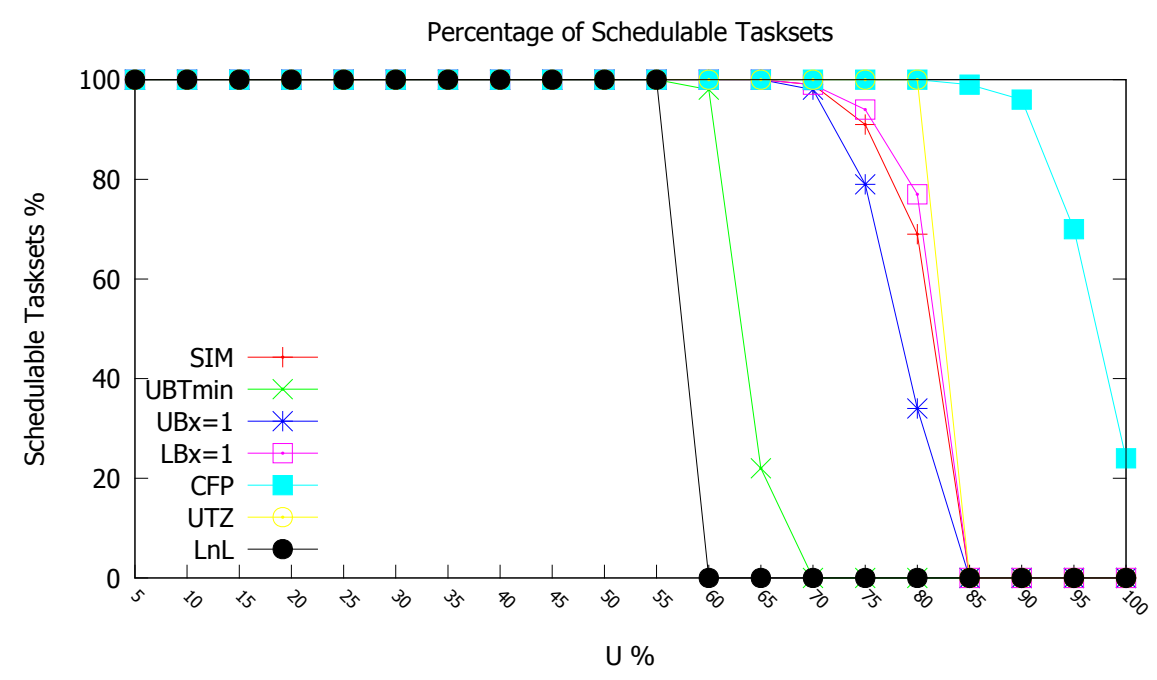

Figure 9: Percentage of Task sets that pass the test over $U$ variation

Figure 9 shows how the percentage of task sets that are deemed schedulable by each of the tests varies with processor utilization. The CFP test has notionally the highest performance since it is widely optimistic and ignores thermal considerations. When temperature is considered, $U T Z, L B_{x=1}$ provide necessary tests, upper bounding the number of task sets that are proved to be schedulable by the exact empirical test $S I M$. We observe that the results confirm that $U B_{T_{\min }}$ and $U B_{x}$ provide sufficient schedulability tests and that for $x=1, U B_{x}$ is tighter bound than $U B_{T_{m i n}}$, with a larger improvement at higher utilization levels. Furthermore, this experiment confirms also the validity of the utilization bounds given in Section 5.2.5 and 5.2.5. With the considered thermal parameters, the maximum achievable utilization for 10 tasks is $80 \%$ for $U T Z$ and the adapted Liu and Layland bound is $57 \%$.

Figure 10 compares the pessimism of $U B_{T_{\min }}$ based shcedulability test to $U B_{x}$ 's one by varying the $x$ parameter. We observe that $U B_{x}$ stays less pessimistic than $U B_{T_{\min }}$ for small values ( $1 \leq x \leq<14$ in this experiment), however, it becomes more pessimistic starting from $x=14$. This result is as expected because the loner cooling periods are, the slower temperature decreases and the longer response times are. 
Figure 11 shows average deviation of bounds from the exact response time given by simulations over processor utilization. The upper bounds have positive values and lower bounds have negative values (the deviation of $S I M$ is 0 because it it gives the exact response time). We can see that deviation of $U B_{x}, L B_{x=1}$ and $C F P$ are still stable over utilization variation in contrast of $U B_{T_{\min }}$ which behaves badly when utilization goes high. We notice also that when $x=1, U B_{x}$ and $L B_{x=1}$ are very close to the actual response time which makes them very interesting tools for approximate schedulability analysis. However, increasing $x$ leads $U B_{x}$ to be less precise, when $x>14, U B_{x}$ behaves as bad as $U B_{T_{\min }}$ or worse.

We also perform further set of experiments showing how schedulability depends on different parameters, including deadlines model and the number of tasks, via the Weighted Schedulability Measure [6] (see our technical report [9]). The conclusions are the following. By varying relative deadlines, we observe that all of the schedulability tests are influenced by the tightness of deadlines to a similar degree, with heavily constrained deadlines having significant impact on schedulability in all cases. We observe also that when we measure the trade-off between tests rate of schedulability and their overhead, the loss of schedulablity is higher than the gain of overhead.

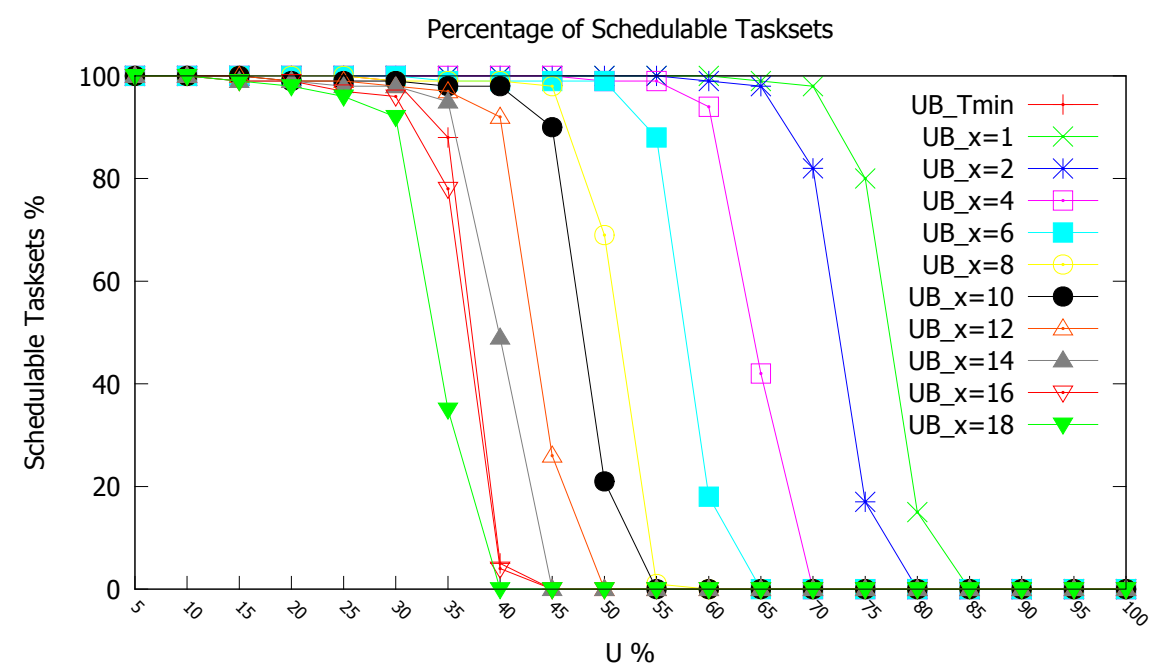

Figure 10: Schedulability by Varying $x$

\section{Conclusion}

In this paper, we addressed the problem of fixed-priority real-time scheduling for thermal-aware systems, where both time and thermal constraints have to be met. Previous research showed that the scheduling policy $P F P_{A S A P}$ is optimal among all fixed-priority scheduling algorithms for non-concrete energy- 


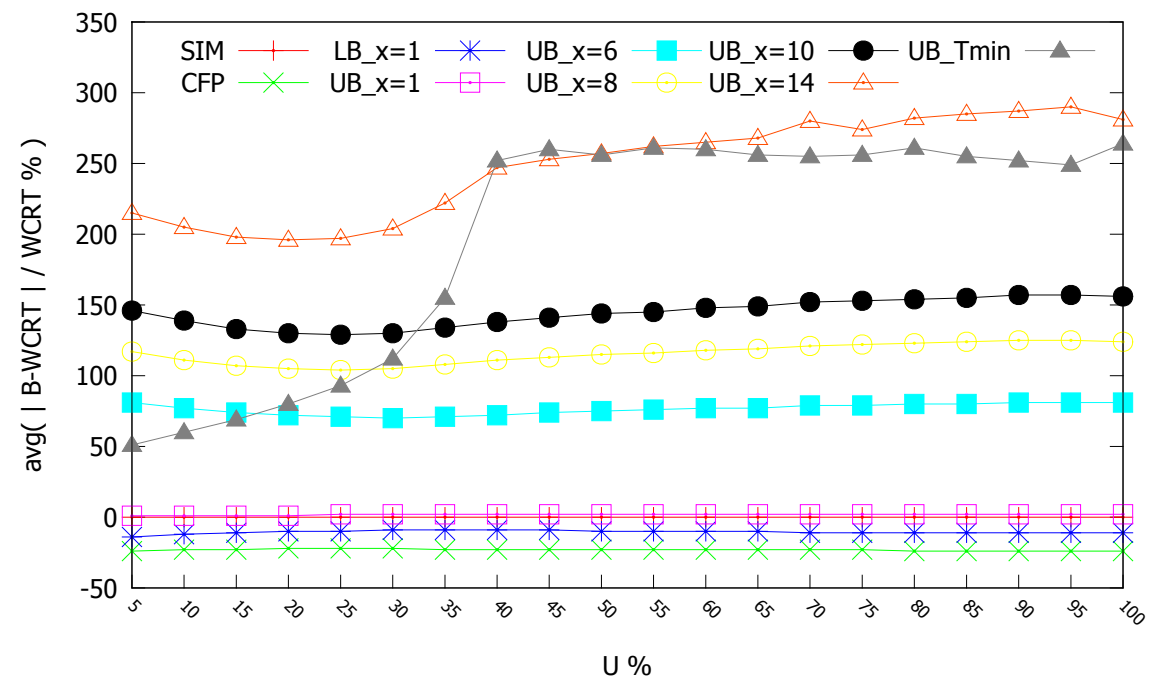

Figure 11: Bounds tightness over U variation

harvesting systems. The main contributions of this paper are as follows: we adpated $P F P_{A S A P}$ algorithm to the thermal-aware model, we proved its optimality and we proposed two schedulability tests based on response-time upper bounds $U B_{T_{m i n}}$ and $U B_{x}$ which is a parametric bound. Finally we performed simulations to validate the theoretical results. As future work, we plan to study deeply the possible similarities between energy-harvesting model and the thermal-aware's one and to explore more adaptable and extensible results of each model.

\section{Acknowledgments}

This research has been supported in part by the US National Science Foundation via a CAREER Grant (CNS-0953585) and a CRI Grant (CNS-1205338).

\section{References}

[1] Yasmina Abdeddaïm, Younès Chandarli, and Damien Masson. The Optimality of PFP asap Algorithm for Fixed-Priority Energy-Harvesting RealTime Systems. In ECRTS, 2013.

[2] Masud Ahmed, Nathan Fisher, Shengquan Wang, and Pradeep Hettiarachchi. Minimizing peak temperature in embedded real-time systems via thermal-aware periodic resources. In SCIS, 2011.

[3] Rehan Ahmed, Parameswaran Ramanathan, and K. Saluja Kewal. On thermal utilization of periodic task sets in uni-processor systems. In RTCSA, 2013. 
[4] Rehan Ahmed, Parameswaran Ramanathan, and K. Saluja Kewal. Necessary and sufficient conditions for thermal schedulability of periodic realtime tasks. In ECRTS, 2014.

[5] André Allavena and Daniel Mossé. Scheduling of frame-based embedded systems with rechargeable batteries. In WPMRTES (with RTAS), 2001.

[6] Andrea Bastoni, BjẪurn B. Brandenburg, and James H. Anderson. Cacherelated preemption and migration delays: Empirical approximation and impact on schedulability. In OSPERTA (with RTAS), 2010.

[7] Enrico Bini and Giorgio C. Buttazzo. Measuring the Performance of Schedulability Tests. RTS, 2005.

[8] Younès Chandarli, Frédéric Fauberteau, Damien Masson, Serge Midonnet, and Manar Qamhieh. YARTISS : A Tool to Visualize, Test, Compare and Evaluate Real-Time Scheduling Algorithms. In WATERS, 2012.

[9] Younès Chandarli, Nathan Fisher, and Damien Masson. Approximate response time analysis for thermal-aware real-time systems under fixed-priority scheduling and reactive control. http://hal. archives-ouvertes.fr/hal-01016114, 2014.

[10] Jian-Jia Chen, Chia-Mei Hung, and Tei-Wei Kuo. On the minimization fo the instantaneous temperature for periodic real-time tasks. In RTAS, 2007.

[11] Jian-Jia Chen, Shengquan Wang, and Lothar Thiele. Proactive speed scheduling for real-time tasks under thermal constraints. In RTAS, 2009.

[12] Pradeep M. Hettiarachchi, Nathan Fisher, Masud Ahmed, Le Yi Wang, Shinan Wang, and Weisong Shi. The design and analysis of thermal-resilient hard-real-time systems. In RTAS, 2012.

[13] LaManna Joseph C., McCracken Kimberly A., Patil Madhavi, and Prohaska Otto J. Stimulus-activated changes in brain tissue temperature in the anesthetized rat. In Metabolic Brain Disease, pages 225-237, 1989.

[14] G. Lazzi. Thermal effects of bioimplants. In EMBM, 2005.

[15] C.L. Liu and James Layland. Scheduling algorithms for multiprogramming in a hard-real-time environment, 1973.

[16] C. Macq and J. Goossens. Limitation of the hyper-period in real-time periodic task set generation. In ICRTS, 2001.

[17] Clemens Moser, D Brunelli, L Thiele, and L Benini. Real-time scheduling with regenerative energy. In ECRTS, 2006.

[18] Gang Quan and Yan Zhang. Leakage aware feasibility analysis for temperature-constrained hard real-time periodic tasks. In ECRTS, 2009. 
[19] Gang Quan, Yan Zhang, William Wiles, and Pei Pei. Guaranteed scheduling for repetitive hard real-time tasks under the maximal temperature constraint. In CODES+ISSS, 2008.

[20] P.S. Ruggera, D.M. Witters, G. von Maltzahn, and H.I. Bassen. In vitro assessment of tissue heating near metallic medical implants by exposure to pulsed radio frequency diathermy. In Physics in Medicine and Biology, pages 2919-2928, 2003.

[21] Kevin Skadron, Mircea R. Stan, Karthik Sankaranarayanan, Wei Huang, Sivakumar Velusamy, and David Tarjan. Temperature-aware microarchitecture: Modeling and implementation. TACO, 2004.

[22] Kim Sohee, Tathireddy P., Normann R.A., and Solzbacher F. Thermal impact of an active 3 -d microelectrode array implanted in the brain. In TNSRE, 2007.

[23] Shengquan Wang, Youngwoo Ahn, and Riccardo Bettati. Schedulability analysis in hard real-time systems under thermal constraints. RTS, 2010.

[24] Shengquan Wang and Riccardo Bettati. Delay analysis in temperatureconstrained hard real-time systems with general task arrivals. In RTSS, 2006.

[25] Shengquan Wang and Riccardo Bettati. Reactive speed control in temperature-constrained real-time systems. RTS, 2008. 